\title{
ARTICLE
}

Cite this: DOI: $10.1039 / \times 0 x \times 00000 x$

\section{The microenvironment of double emulsions in rectangular microchannels}

\author{
Shaohua Ma, ${ }^{a, b}$ Joseph M. Sherwood, ${ }^{c, d}$ Wilhelm T. S. Huck, ${ }^{a, e}$ and Stavroula \\ Balabani $^{\mathrm{C}}$
}

Received ooth January 2015,

Accepted ooth January 2015

DOI: $10.1039 /$ xoxxooooox

The flow environment in inner cores of water-in-oil-in-water (w/o/w) microfluidic double emulsions has a significant impact on industrial applications of such systems. For example, in the case of shear sensitive cells compartmentalised in the cores, high shear conditions may be deleterious. This study reports on the flow characteristics of $\mathrm{w} / \mathrm{o} / \mathrm{w}$ inner cores in comparison to those in single water-in-oil (w/o) microdroplets of equal size moving in the same microchannel, resolved by means of micro-particle image velocimetry ( $\mu \mathrm{PIV})$. The multiphase flow system employed in the study had a viscosity ratio, $\lambda$, between aqueous and oil phase of the order of unity $(\lambda=0.78)$ and both single droplets and inner cores of double emulsions filled the channel. This configuration resulted in a weak recirculating flow inside the w/o single droplet: the measured flow field exhibited a uniform low velocity flow field in the central region surrounded by small regions of reversed flow near the channel walls. This flow topology was maintained in the inner cores of $\mathrm{w} / \mathrm{o} / \mathrm{w}$ double emulsions for intermediate capillary numbers $(\mathrm{Ca})$ ranging from $10^{-3}$ to $10^{-2}$, and core morphologies varying from large plugs to pancake cores. The core morphology affected the magnitude and distribution of the velocity in the droplets. The similarity in the flow topology resulted from the fact that inner cores were located at the back of the outer droplet in such a way that inner and outer interfaces were in contact for over half of core surface area and separated only by a thin lubricating film. Both single droplets and inner cores exhibited a narrow shear rate distribution characterised by small regions of maximum shear confined near the channel walls. Shear rate magnitude values were found to be an order of magnitude lower than those in the channel and hence capable of reducing stresses in flow cytometry to far below reported values for cell damage. Hence, it can be concluded that double emulsions are suitable candidates to substitute single droplets in flow cytometry to protect the screened items and are compatible with the commercial flow cytometry systems. 


\section{Introduction}

Droplet-based microfluidics has been the subject of extensive research due to the wide ranging applications of this technology, as it minimises the volume and time scale required in micromanipulation, by compartmentalising targets in droplets and isolating them in the continuous phase. ${ }^{1}$ Knowledge of the flow field inside the droplets is paramount in order to tailor droplet-based microfluidics to a specific application. W/o/w double emulsions have advantages over single w/o droplets in the formulation of complex microparticles ${ }^{2,3}$ and giant polymersomes ${ }^{4-7}$, targeted in controlled drug release $^{8-12}$ as well as biomimetic cellular structures ${ }^{13-}$ 15. High-throughput cell manipulation requires compartmentalisation at high speed, which generates a harsh shear environment inside the droplets, potentially harming the viability of certain cells. ${ }^{16}$ For example, strong shear forces, originating from the hydrodynamic focusing effect that aligns the cell samples flowing through the nozzle into a single file flow, have been found to cause physiological alterations and reduce fertilisation after gender sorting of sperm cells ${ }^{17-19}$ or cause dysfunction on some stem cells ${ }^{20}$ in flow cytometry. Droplet screening offers a route to protect sensitive cells against such external shear stresses in flow cytometry, on the condition that the droplet interior provides a milder microenvironment to the screened cells. However, w/o droplets are not compatible with commercial flow cytometry methods, such as fluorescence-activated cell sorting (FACS), owing to the fact that the carrier fluid in FACS is aqueous, whereas w/o droplets are carried by oil. Monodisperse w/o/w double emulsions have been proposed to overcome this limitation and been demonstrated as a flow cytometry-compatible screening platform. ${ }^{21}$ However, in order to take the technology further, knowledge of the flow conditions in the inner aqueous cores of $\mathrm{w} / \mathrm{o} / \mathrm{w}$ double emulsions is required, which provides the motivation for the present study.

Micro-particle image velocimetry ( $\mu$ PIV) can be used to quantitatively characterize microscale flow fields ${ }^{22}$, including those in single droplets and double emulsions. In recent years, it has been widely used to study the droplet flow topology of liquid-liquid systems in microfluidics. ${ }^{23-29}$ However, to the best of our knowledge no $\mu$ PIV study has reported on the flow conditions in double emulsions, especially those in inner cores of w/o/w double emulsions.

The present study reports on the flow characteristics inside the aqueous inner cores of w/o/w double emulsions, comprising two aqueous phases and one fluorinated oil phases (with surfactants added in selected phases), flowing in a rectangular microchannel. The velocity field measured with $\mu \mathrm{PIV}$ in the inner aqueous cores was compared to that found in aqueous single droplets of approximately the same size, in the $C a$ range $10^{-3}$ to $10^{-2}$.

\section{Experimental}
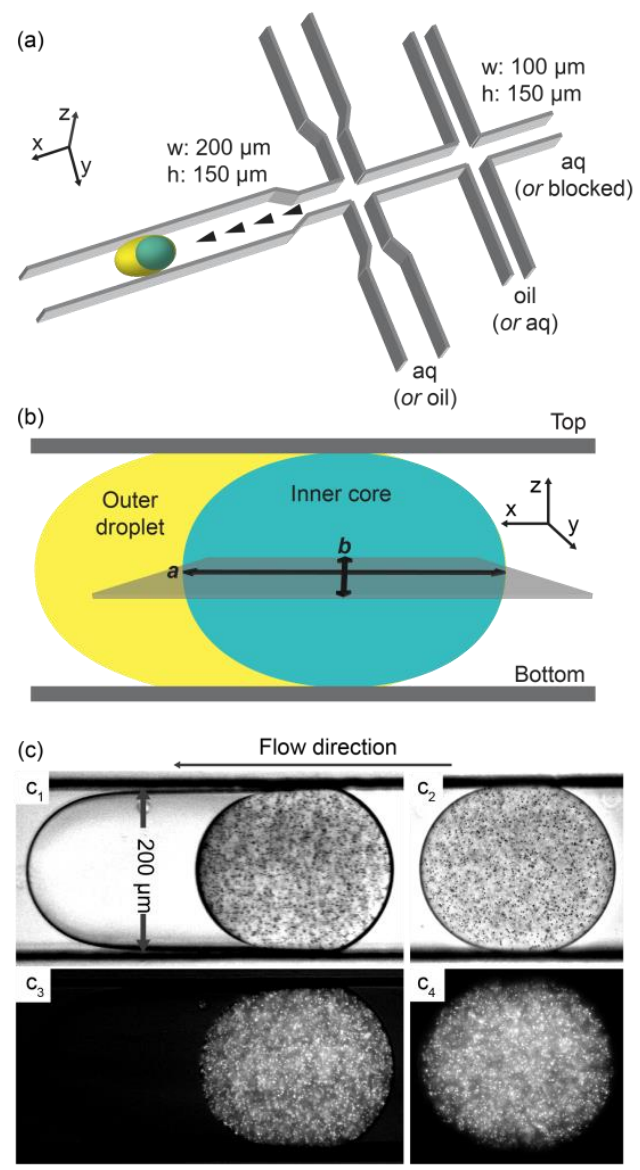

Fig. 1 Formation and image acquisition of double emulsions and single droplets. (a) 3D drawing of the microfluidic channel architecture. The channel has a fixed depth of $150 \mu \mathrm{m}$; the channel width is $100 \mu \mathrm{m}$ in the formation region and $200 \mu \mathrm{m}$ further downstream as illustrated. Single droplets are generated by blocking the innermost inlet channel (w/o configuration given in the brackets). The long straight channel downstream of the second flow focusing junction, where droplet images are acquired, is 20 $\mathrm{mm}$ long. (b) Illustration of the central plane across the inner core where images are acquired. The dimensions of the inner core in $x, y$ directions are denoted $a$ and $b$, respectively. (c) Typical $\left(c_{1}, c_{2}\right)$ bright-field and $\left(c_{3}, c_{4}\right)$ fluorescent images of $\left(c_{1}, c_{3}\right) w / o / w$ double emulsions and $\left(c_{2}, c_{4}\right)$ w/o droplets explored in this paper.

All chemicals were purchased from Sigma Aldrich Co. UK, unless noted otherwise and used without further purification. Deionised water was used in all aqueous phases. Microfluidic PDMS devices were fabricated by combined photo- and softlithography, ${ }^{30}$ and have been described in detail previously. ${ }^{29}$ A channel depth of $150 \mu \mathrm{m}$ was achieved by spin-coating SU8 2025 (MicroChem, USA) at $75 \mu \mathrm{m}$ onto a 3 -inch silicon wafer (Compart Technology Ltd, UK) twice. The microchannel geometry is shown in Fig. 1a. The width of the 
channels was $100 \mu \mathrm{m}$ upstream of the second flow focusing junction and $200 \mu \mathrm{m}$ downstream. The channel surfaces were made hydrophobic for w/o droplet formation by flushing the channel with $0.5 \%$ Trichloro $(1 H, 1 H, 2 H, 2 H$-perfluorooctyl) silane in HFE 7500 (297730-93-9, 3M, 0.77cSt). Selective surface modification ${ }^{31}$ was adopted to form w/o/w double emulsions, by depositing polyelectrolytes layer-by-layer and flushing silane in selected regions, sequentially.

The aqueous and the oil phases were injected independently into the microchannel. The inner aqueous fluid (1.0 M phosphate buffered saline (PBS), $\mathrm{pH}$ 7.4) formed w/o droplets at the first crossjunction by flow-focusing with the fluorinated oil HFE 7500 (297730-93-9, 3M, USA), loaded with 1.5 wt\% Pico-Surf ${ }^{\mathrm{TM}} 1$ surfactant (Sphere Fluidics Ltd., UK), and the secondary emulsification of these droplets followed at the second cross-junction, generating w/o/w double emulsions. The continuous phase was water, loaded with 0.5 wt $\%$ sodium dodecyl sulphate (SDS). The inner core phase was seeded with neutrally buoyant $1.1 \mu \mathrm{m}$ Nile Red fluorescent tracing particles (FluoSphere F-8819, Invitrogen Inc., USA) at a concentration of $6 \times 10^{6}$ particles per $\mathrm{ml}$ before flow delivery. The volume fraction of these seeding particles in flow was only 4 $\times 10^{-6}$, and hence no effect on the measured flow topology was expected. Single droplets were formed in the same microfluidic device by blocking the innermost inlet. As both the inner cores and single droplets studied filled the channel width, monodispersity of inner cores and single droplets was established by their length distribution which showed a coefficient of variation less than $3 \%$ in all cases. As the flow topology in single droplets and inner cores of double emulsions at equilibrium is not time-dependent, ensemble correlation of multiple image pairs significantly reduces topology errors.

A $\mu$ PIV system developed by Sherwood et al. ${ }^{32}$ was used for measurements of the flow field inside the droplets. ${ }^{29}$ The microfluidic device was mounted onto the stage of an inverted microscope (Leica DM ILM, Germany). The (inner) aqueous and oil phases were pumped into the microchannel at various flow rates with a syringe pump (Fusion 400, Chemyx Inc., USA), and the continuous aqueous phase in the double emulsions was perfused from a pressure controlled aqueous reservoir. ${ }^{33}$ Fluorescent and strobe images were acquired alternatively using a CCD camera (C8484-05C, Hamamatsu, Japan) operating in double-frame mode at $6 \mathrm{~Hz}$. A $10 \times$ (NA $=0.25$ ) objective lens was used to acquire double emulsion images, whereas single droplet images were acquired by a $20 \times(\mathrm{NA}=0.40)$ lens, with depth of correlation (DOC) of $38 \mu \mathrm{m}$ and $15 \mu \mathrm{m}$, respectively. ${ }^{34}$
The different DOC will result in different levels of underestimation in the reported velocities, but as both objectives give DOC less than a third of the channel depth, the observed flow topologies are unlikely to be affected. This was verified by analysing a single droplet with both objectives (not shown for brevity).

Triggering and image acquisition were carried out via Labview (National Instrument, Texas, USA). Fig. $1 \mathrm{~b}$ illustrates the central focal plane, where images were acquired. Fig. 1c shows typical bright-field and corresponding fluorescent images for a double emulsion $\left(\mathrm{c}_{1}, \mathrm{c}_{3}\right)$ and a single droplet $\left(\mathrm{c}_{2}, \mathrm{c}_{4}\right)$.

$\mathrm{Ca}$ was defined based on the velocity of the continuous phase, $v_{c}$, as

$$
C a=\mu_{c} v_{c} / \gamma
$$

where $\mu_{c}$ is the viscosity of the continuous phase, and $\gamma$ is the w/o interfacial tension. The viscosity ratio of the aqueous phase against the oil phase, $\lambda$, is of the order of unity $(\lambda=0.78)$ and both single droplets and inner cores of double emulsions filled the channel. As the surfactants (Pico-Surf ${ }^{\mathrm{TM}} 1$ or SDS) are used at very low concentrations in the oil or outer aqueous phase, we assume the viscosity is unaltered by the surfactant. The inner cores were found to be located at the rear of the double emulsions, when the flow reached an equilibrium state, and did not move relative to the oil shell. The distortion resulting from the mismatch in the refractive indices of water (1.33, $\left.20{ }^{\circ} \mathrm{C}\right)$ and HFE $7500\left(1.29,20{ }^{\circ} \mathrm{C}\right)$ in the water droplets in HFE 7500 was found to be lower than $5 \%$, according to a ray tracing methodology. ${ }^{35}$

\section{Results and Discussion}

Figures $2 \mathrm{a}-2 \mathrm{c}$ show velocity vectors in the central plane of w/o/w double emulsions with different sized inner cores $(a / b=1.2-2.2)$ and Fig. $2 \mathrm{~d}$ shows velocity vectors of a small plug single droplet $(a / b=$ 1.3); all vectors have been normalised by the velocity of the inner cores or the single droplet, $v_{d}$. The flow topology is similar in all cases: the central region exhibits low magnitude, near zero, velocity, accompanied by two regions of relative high velocity, reversed flow (i.e. opposite to the droplet motion) on both sides of the centreline and close to the w/o interfaces.

Our previous study on single droplets ${ }^{29}$ attributed this flow topology to the viscosity ratio $\lambda$ of the liquid-liquid system employed. The viscosity ratio was found to be the key factor that determines the droplet flow topology and in particular the extent of recirculation, for the range of $\mathrm{Ca}$ studied. The less viscous fluorinated oil phase employed yields $\lambda=$ 0.78 across the w/o interface of single droplets, resulting in velocity gradients of similar magnitude and hence less flow reversal along the droplet centreline. In the w/o/w double emulsion system, 


\section{ARTICLE}
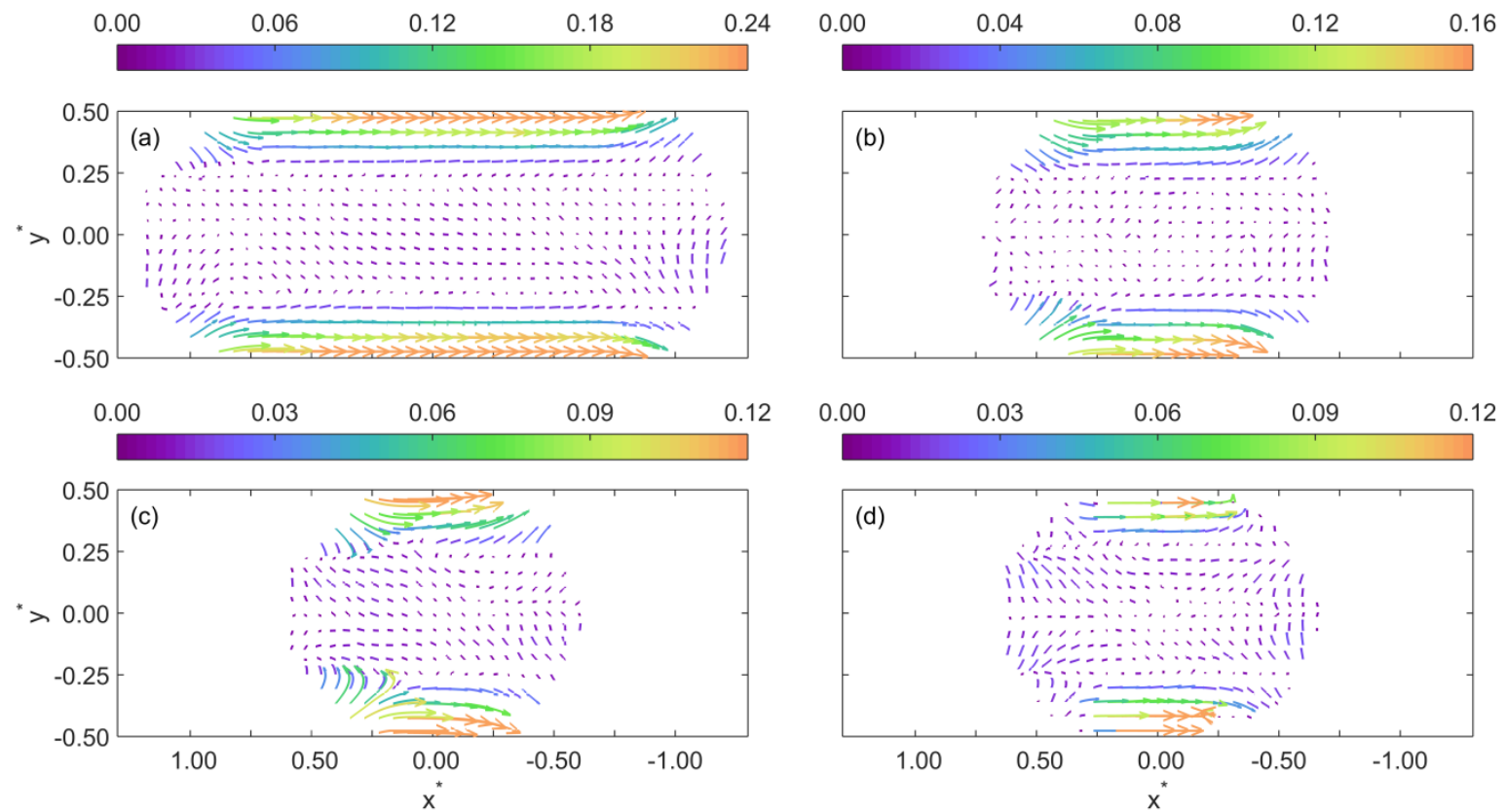

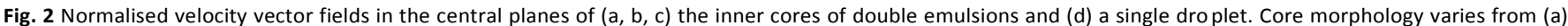

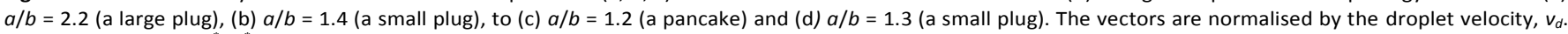

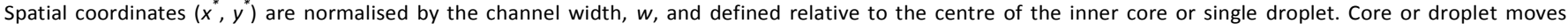
towards left. Ca: (a) $1.9 \times 10^{-3}$; (b) $7.1 \times 10^{-3}$; (c) $13.2 \times 10^{-3}$; (d) $6.9 \times 10^{-3}$.

two liquid-liquid interfaces are encountered, w/o (water-in-oil) and $\mathrm{o} / \mathrm{w}$ (oil-in-water), with corresponding viscosity ratios of 0.78 and 1.28 , both in the order of unity. The inner core is pushed to the rear of the shell by drag, and there is no relative motion between the inner core and double emulsion itself and only a thin oil film separates the two which is marginally more viscous (1.28 times) than the aqueous film between the emulsion and the channel walls. The combination of these two effects can explain the similar topologies observed. It was also found that the measured flow topology persisted for $\mathrm{Ca}$ ranging from $10^{-3}$ to $10^{-2}$.

Figure 3 compares the axial and transverse velocity distributions measured in double and single droplets with aspect ratios of 1.4 and 1.3 respectively. The velocities are presented in the reference frame of the droplet and are normalised by the droplet velocity $v_{d}, \bar{u} / v_{d}$ and $\bar{v} / v_{d}$, respectively. The distributions of both components, $\bar{u} / v_{d}$ and $\bar{v} / v_{d}$, are qualitatively similar; axial velocities reach similar magnitude values, whereas the transverse velocities are higher in the inner core than in the single droplet, which can possibly be attributed to the slight mismatch in the aspect ratio, and / or the contribution of the oil plug shell in the double emulsion. The distributions of the two velocity components were found to be similar among cores with aspect ratios $a / b$ ranging from 1 to 2.5. As described previously, the similarity can be attributed primarily to the thin oil film separating the aqueous inner core and the outer oil shell, and the morphology of the double emulsion; over half of the w/o interface coincides with the o/w interface, due to the inner cores filling the entire channel width in this study. Although the w/o interface is not totally coincident with the $\mathrm{o} / \mathrm{w}$ interface in the double emulsions, the common part appears to primarily dictate the topology observed. The thin oil film and the viscosity ratio of the aqueous core to the oil shell $(\lambda=0.78)$, in conjunction with the velocity and stress continuity condition at the interface (assuming uniform interfacial tension) result in axial velocities of similar magnitude across the $w / o$ and $\mathrm{o} / \mathrm{w}$ interfaces, i.e. from the inner core to the continuous phase. 


\section{ARTICLE}
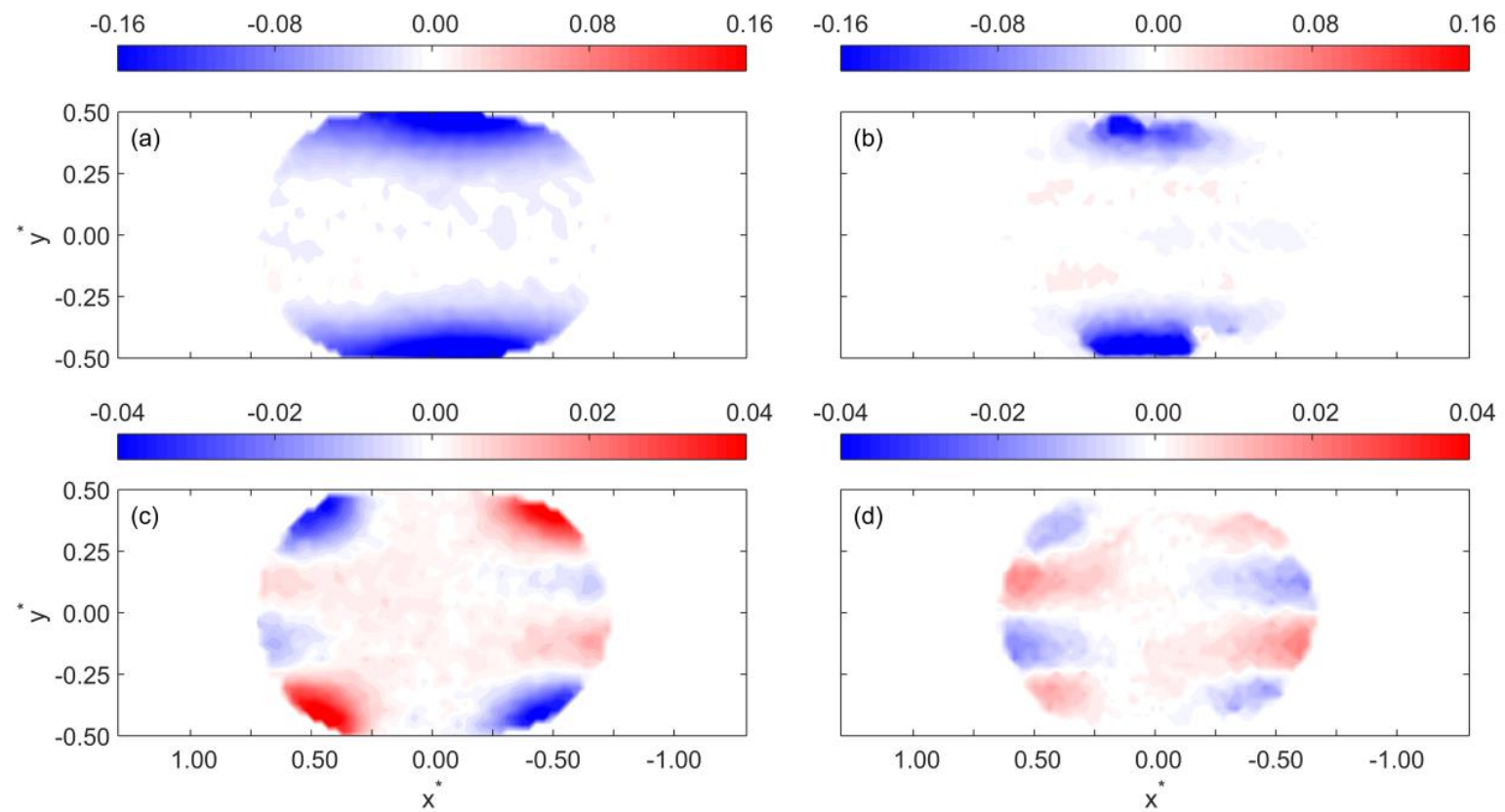

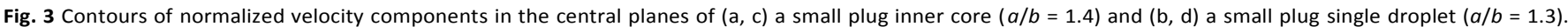
The velocity components are normalised by the droplet velocity, i.e. (a, b) $\bar{u} / v_{d}$ and $(\mathrm{c}, \mathrm{d}) \bar{u} / v_{d} . \mathrm{Ca}:(\mathrm{a}, \mathrm{c}) 7.1 \times 10^{-3},(\mathrm{~b}, \mathrm{~d}) 6.9 \times 10^{-3}$.

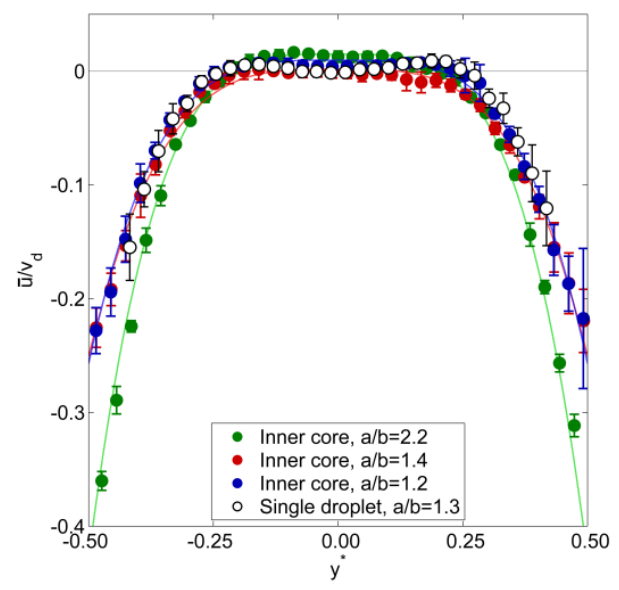

Fig. 4 Profiles of $\bar{u} / v_{d}$ at $x^{*}=0$ for each of the droplets shown in Fig. 2. Error bars show 2 standard deviations. Dashed lines are fits to $\bar{u} / v_{\mathrm{d}}=b-u_{\max }\left(1-\left|x^{a}\right|\right)$.

To allow a better comparison between the measured velocities, axial velocity profiles $\bar{u} / v_{d}$ across the droplet and at $x^{*}=0$ are plotted in Fig. 4. The profiles further demonstrate the flow similarity between inner cores and single droplets of similar size. The low velocity region in the droplet interior $\left(-0.25<y^{*}\right.$
$<0.25)$ persists in all configurations; however, the inner core of the large plug double emulsion $(a / b=2.2)$ shows a steeper gradient near the interface compared to the smaller cores $(a / b=$ 1.4 and 1.2) and this is accompanied by low positive velocities in the central part.

The present study shows that inner cores of w/o/w double emulsions exhibit similar flow patterns as equally sized w/o single droplets - in the specific eccentric configuration studied. The core geometry and $\mathrm{Ca}$ (in the range considered) affect only the magnitude of flow velocities, as was shown in Figs. 2 and 4, with the axial velocity approaching channel walls $\left(0.25<\left|y^{*}\right|<\right.$ $0.5)$ nearly doubling as the core size increased from $a / b=1.2$ to $a / b=2.2$. In the $C a$ range studied, the Bond number, $B o=$ $\Delta \rho g l^{2} / \gamma$, that represents the ratio of gravitational to surface tension forces, is far below 1 ( $\Delta \rho$ is the difference in fluid densities, $g$ is the acceleration of gravity, $l$ is a characteristic length scale, and $\gamma$ is the interfacial tension of two fluids). ${ }^{36}$ As gravitational forces do not have dominant effect in microfluidics, it is reasonable to assume symmetry and expect the core flow topology to vary accordingly in the $y z$ plane, from which we can conclude that the central region of the inner cores exhibits low velocities, whereas the regions near the channel boundaries have higher magnitude and reversed velocity flows. 


\section{ARTICLE}
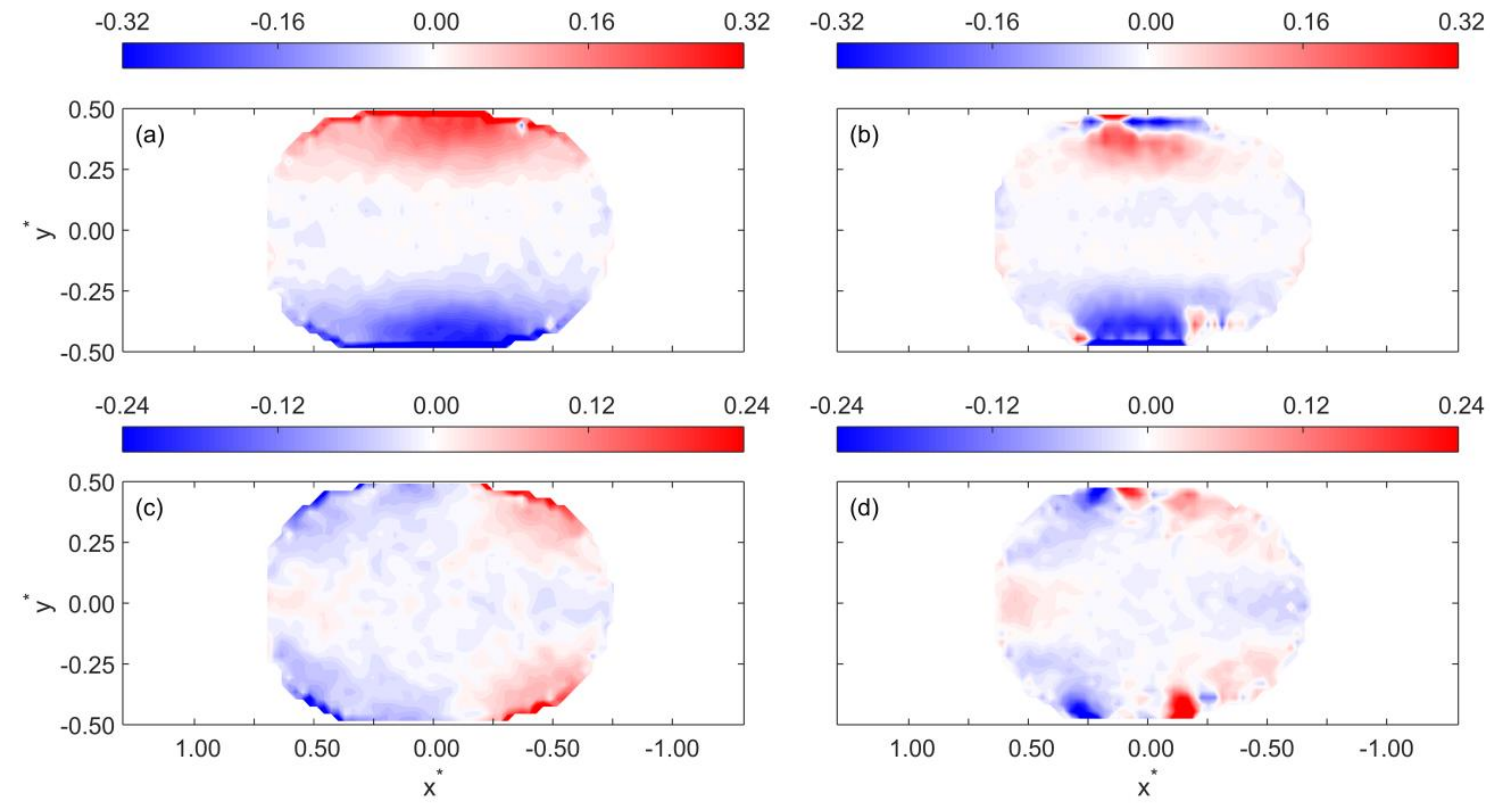

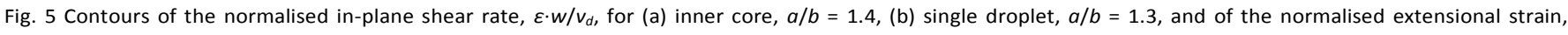
$\eta \cdot w / v_{d}$, for (c) inner core, $a / b=1.4$, (d) single droplet, $a / b=1.3$.

In order to evaluate the shear environment of double emulsions for cell encapsulation applications, the extensional and shear strain rates were estimated from the measured velocity fields. Hydrodynamic stresses act in all three dimensions and hence accurate estimation of the shear rate magnitude requires both elongational and shear strain terms to be accounted for, i.e. ${ }^{37}$

$|\dot{\gamma}|=\left[\begin{array}{c}2\left(\frac{\partial u}{\partial x}\right)^{2}+2\left(\frac{\partial v}{\partial y}\right)^{2}+2\left(\frac{\partial w}{\partial z}\right)^{2}+\left(\frac{\partial v}{\partial x}+\frac{\partial u}{\partial y}\right)^{2}+ \\ \left(\frac{\partial w}{\partial y}+\frac{\partial v}{\partial z}\right)^{2}+\left(\frac{\partial u}{\partial z}+\frac{\partial w}{\partial x}\right)^{2}\end{array}\right]^{\frac{1}{2}}$

This can only be achieved if the velocity gradient tensor $\nabla \boldsymbol{U}$ is fully resolved, i.e. all nine velocity gradients are quantified.

In the current $2 \mathrm{D} \mu \mathrm{PIV}$ study, however, only four of these gradients could be estimated. Based on these gradients, the inplane shear and extensional strain rates were calculated from $\varepsilon$ $=\partial \bar{u} / \partial y+\partial \bar{v} / \partial x$ and $\eta=\partial \bar{u} / \partial x+\partial \bar{v} / \partial y$, respectively. The velocity gradients were estimated from the measured velocities using the least squares method. ${ }^{38}$ Caution is required in interpreting the strain values as differencing introduces some errors that depend on the scheme used. Strain rate values are normalised by a nominal shear rate given by $v_{d} / w$ and are shown in Fig. 5 for both inner cores of double emulsions and similarly sized single droplets with aspect ratios as in Fig. 3.

As expected, both the in-plane shear rate (Figs. 5a, b) and the extensional strain (Figs. 5c, d) exhibit similar distributions between the inner core (Figs. 5a, c) and the single droplet (Figs. $5 b, d)$. The maximum values of the in-plane shear rate and extensional strain are approximately equal to $48 \mathrm{~s}^{-1}$ and $36 \mathrm{~s}^{-1}$, which correspond to stress values of $48 \mathrm{mPa}$ and $36 \mathrm{mPa}$, respectively. In the absence of more accurate information on the full stress tensor and the location of cells within the droplet, it is reasonable to assume that the estimated values provide an indication of the magnitude of the maximum stresses encapsulated cells are likely to experience inside the droplets (either single or double).

Hydrodynamic shear is an important parameter for cell viability; various ways to correlate cell damage to flow have been discussed in the literature with the stress tensor or the energy dissipation rate (EDR) been most commonly used. ${ }^{39}$ Cells are likely to experience a range of hydrodynamic stresses during encapsulation or in bioprocessing in general. Sensitivity to these factors is cell specific and hence it is difficult to ascertain whether the stress values measured inside the droplets can ensure cell viability for any application. For example, stress values ranging from 0.02 to $2 \mathrm{~Pa}$ have been reported for the 


\section{ARTICLE}
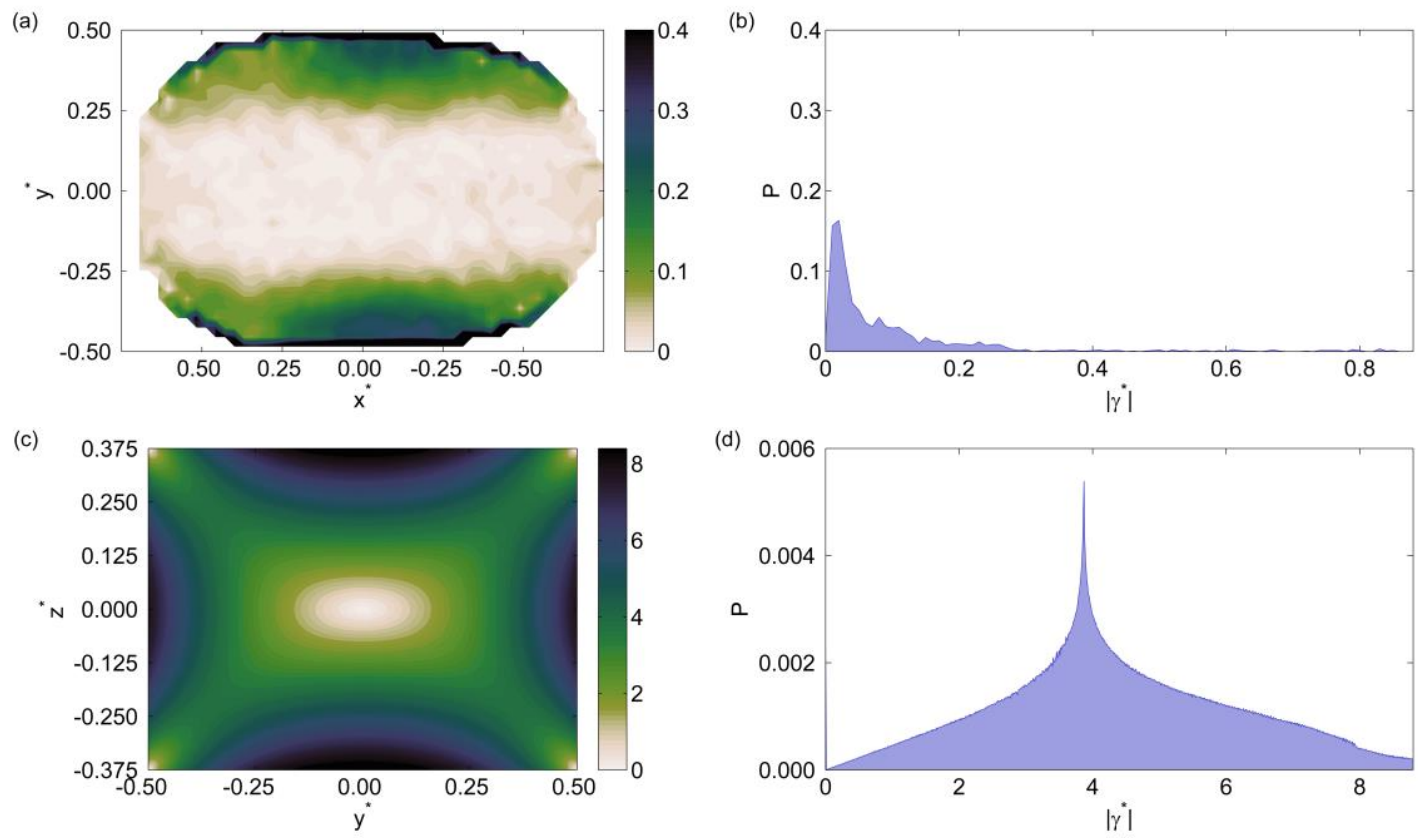

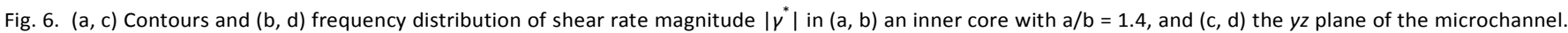
Values are normalised by the nominal shear, $v_{c} / w$.

differentiation of stem cells. ${ }^{40}$ It should be noted however that both the magnitude of hydrodynamic forces as well as the exposure length are important factors for cell viability.

In order to further elucidate the benefits of droplet cell encapsulation, a comparison between the stress environment inside an inner core plug to that in the microchannel flow is provided in Fig. 6. Using equation (1) and omitting the missing velocity gradients (due to $3^{\text {rd }}$ velocity component or gradients in the $z$-direction), the shear rate magnitude $\left|\gamma^{*}\right|$ distribution in the $x y$ plane of the inner core is obtained (Fig. 6a) and compared to that in the $y z$ plane of the microfluidic channel (Poiseuille) flow (Fig. 6c) obtained using the analytical solution of Bruus. ${ }^{41}$ Values are normalised by the nominal shear $\left(v_{c} / w\right)$. Histograms of the shear rate magnitude for both geometries are also shown in Figs. $6 b$ and d. It can be seen that in the present study droplets result in a narrow distribution of shear rate magnitude values compared to the microfluidic channel flow with a mean value which is an order of magnitude lower. Even if the additional gradient terms are included the shear in the droplet will remain significantly lower than that in the channel. Very few studies have dealt with cell damage in flow cytometry applications. Mollet et al. reported significant cell death rates for certain cell types in FACS at EDR over $10^{7} \mathrm{~W} / \mathrm{m}^{3}$, corresponding to shear rates approximately $10^{3}-10^{4} \mathrm{~s}^{-1}$. ${ }^{42}$ The maximum dissipation rates were observed in the contraction region of the sheath flow. By taking into account that shear rates inside the droplets were found to be an order of magnitude lower than those in the microchannel flow, it can be expected that the measured stresses are significantly lower than the lethal range for most cell types. It is likely that the magnitude of shear at the actual flow focusing junction, encapsulation point, is higher than the values found inside droplets. There is also evidence that elongational stresses are more harmful to cells than pure shear and hence further investigation is needed to quantify the maximum shear exposure of cells and the suitability of double emulsion in FACS. Furthermore the stability of the double emulsions is of importance and shear deformation studies or studies in which all phases are simultaneously resolved will be useful.

\section{Conclusion}

The flow field in the inner cores of w/o/w double emulsions flowing in a rectangular microchannel was studied by means of $\mu$ PIV. An identical flow topology to that of single droplets was observed at moderate $\mathrm{Ca}, 10^{-3}$ to $10^{-2}$. In the liquid-liquid systems employed, low velocity flow was found in the central droplet region and high velocity reversed flow was confined 
along the interface, unlike the typical recirculating topology reported in previous studies; this was due to the viscosity ratio in the present study as a less viscous, biocompatible oil was employed. The study shows that $\mathrm{Ca}$ and core (droplet) morphology do not affect the flow topology, but affect the velocity distribution and magnitude. The inner cores exhibit analogous flow topologies and velocity magnitudes with equally sized single droplets composed of the inner core and middle oil shell of double emulsions. Furthermore, maximum stresses are confined in a small region near the channel walls and are an order of magnitude lower than those in the channel. The results indicate that double emulsions are suitable candidates to substitute single droplets in flow cytometry to protect the screened items and are compatible with the commercial flow cytometry systems. Thus potentially w/o/w double emulsions can protect screened sperm or stem cells from harsh shear environment in FACS and achieve better fertility rate and reduced stem cells dysfunction rate.

\section{Acknowledgements}

SM acknowledges the financial support provided by the CSC Cambridge Scholarship throughout his $\mathrm{PhD}$ studies. SB would like to thank Dantec Dynamics for the loan of the laser used in this study. Finally the authors wish to thank Dr Peter Vennemann for providing the free PIV analysis software JPIV (www.jpiv.vennemann-online.de).

\section{Notes and references}

a Department of Chemistry, University of Cambridge, Cambridge CB2 $1 \mathrm{EW}, \mathrm{UK}$.

b Chemistry Research Laboratory, University of Oxford, Oxford OX1 3TA, UK.

c Department of Mechanical Engineering, University College London, London WC1E 7JE, UK. Email: s.balabani@ucl.ac.uk.

d Department of Bioengineering, Imperial College London, London SW7 2AZ, UK.

e Radboud University Nijmegen, Institute for Molecules and Materials, Heyendaalseweg 135, 6525 AJ Nijmegen, The Netherlands.

\section{References}

1 A. B. Theberge, F. Courtois, Y. Schaerli, M. Fischlechner, C. Abell, F. Hollfelder, W. T. S. Huck, Angew. Chem. Int. Ed. 2010, 49, 58465868.

2 Z. Nie, S. Xu, M. Seo, P. C. Lewis, and E. Kumacheva, J. Am. Chem. Soc., 2005, 127, 8058-8063.

3 F. Gao, Z.-G. Su, P. Wang, and G.-H. Ma, Langmuir, 2009, 25, 3832-3838.

4 F. Meng, G. H. M. Engbers, and J. Feijen, J. Control. Release, 2005, 101, 187-198.

5 H. C. Shum, J.-W. Kim, and D. A. Weitz, J. Am. Chem. Soc., 2008, 130, 9543-9549.

6 S.-H. Kim, J. Nam, J. W. Kim, D.-H. Kim, S.-H. Han, and D. A. Weitz, Lab Chip, 2013, 13, 1351-1356.

7 J. Thiele, V. Chokkalingam, S. Ma, D. A. Wilson, and W. T. S. Huck, Mater. Horizons, 2014, 1, 96-101.
8 R. Rodríguez-García, M. Mell, I. López-Montero, J. Netzel, T. Hellweg, and F. Monroy, Soft Matter, 2011, 7, 1532-1542.

9 C. LoPresti, H. Lomas, M. Massignani, T. Smart, and G. Battaglia, $J$. Mater. Chem., 2009, 19, 3576-3590.

10 J.-W. Kim, A. S. Utada, A. Fernández-Nieves, Z. Hu, and D. A. Weitz, Angew. Chemie, 2007, 119, 1851-1854.

11 K. T. Kim, J. J. L. M. Cornelissen, R. J. M. Nolte, and J. C. M. van Hest, Adv. Mater., 2009, 21, 2787-2791.

12 S.-H. Kim, H. C. Shum, J. W. Kim, J.-C. Cho, and D. A. Weitz, J. Am. Chem. Soc., 2011, 133, 15165-15171.

13 M. Marguet, L. Edembe, and S. Lecommandoux, Angew. Chemie, 2012, 124, 1199-1202.

14 C. Martino, S.-H. Kim, L. Horsfall, A. Abbaspourrad, S. J. Rosser, J. Cooper, and D. A. Weitz, Angew. Chem. Int. Ed. Engl., 2012, 51, 6416-6420.

15 M. Marguet, C. Bonduelle, and S. Lecommandoux, Chem. Soc. Rev., 2013, 42, 512-529.

16 E. Brouzes, M. Medkova, N. Savenelli, D. Marran, M. Twardowski, J. B. Hutchison, J. M. Rothberg, D. R. Link, N. Perrimon, and M. L. Samuels, Proc. Natl. Acad. Sci. U. S. A., 2009, 106, 14195-14200.

17 T. Leahy and B. M. Gadella, Reproduction, 2011, 142, 759-778.

18 D. Rath, S. Barcikowski, S. De Graaf, W. Garrels, R. Grossfeld, S. Klein, W. Knabe, C. Knorr, W. Kues, H. Meyer, J. Michl, C. Rehbock, U. Taylor, S. Washausen, S. de Graaf, and G. MoenchTegeder, Reproduction, 2013, 145, R15-30.

19 W. Maxwell, G. Welch, and L. Johnson, Reprod. Fertil. Dev., 1996, 8, 1165-1178.

20 L. Williams and Y. Peter, J Organ Dysfunct, 2007, 3, 31-35.

21 J. Yan, W. A. C. Bauer, M. Fischlechner, F. Hollfelder, C. F. Kaminski, and W. T. S. Huck, Micromachines, 2013, 4, 402-413.

22 R. Lindken, M. Rossi, S. Grosse, and J. Westerweel, Lab Chip, 2009, 9, 2551-2567.

23 D. Malsch, M. Kielpinski, R. Merthan, J. Albert, G. Mayer, J. M. Köhler, H. Süße, M. Stahl, and T. Henkel, Chem. Eng. J., 2008, 135S, S166-S172.

24 H. Kinoshita, S. Kaneda, T. Fujii, and M. Oshima, Lab Chip, 2007, 7, 338-346.

25 L. Prat, C. Gourdon, F. Sarrazin, K. Loubie, T. Bonometti, and J. Magnaudet, AIChE J., 2006, 52, 4061-4070.

26 C. King, E. Walsh, and R. Grimes, Microfluid. Nanofluidics, 2007, 3, 463-472.

27 U. Miessner, R. Lindken, and J. Westerweel, in Proc. 14th Int. Symp. on Appl. of Laser Tech. to Fluid Mech., 2008, pp. 7-10.

28 V. Dore, D. Tsaoulidis, and P. Angeli, Chem. Eng. Sci., 2012, 80, 334-341.

29 S. Ma, J. M. Sherwood, W. T. S. Huck, and S. Balabani, Lab Chip, 2014, 14, 3611-3620.

30 Y. Xia and G. M. Whitesides, Annu. Rev. Mater. Sci., 1998, 28, 153184.

31 W.-A. C. Bauer, M. Fischlechner, C. Abell, and W. T. S. Huck, Lab Chip, 2010, 10, 1814-1819.

32 J. M. Sherwood, E. Kaliviotis, J. Dusting, and S. Balabani, Biomech. Model. Mechanobiol., 2014, 13, 259-273.

33 J. M. Sherwood, J. Dusting, E. Kaliviotis, and S. Balabani, Biomicrofluidics, 2012, 6, 024119.

34 M. G. Olsen and R. J. Adrian, Exp. Fluids, 2000, 29, S166-S174. 
35 G. Minor, P. Oshkai, and N. Djilali, Meas. Sci. Technol., 2007, 18, L23-L28.

36 A. Günther and K. F. Jensen, Lab Chip, 2006, 6, 1487-1503.

37 E. Kaliviotis, J. Dusting, and S. Balabani, Med. Eng. Phys., 2011, 33, 824-831.

38 M. Raffel, C. E. Willert, S. T. Wereley, and J. Kompenhans, Particle Image Velocimetry: A Practical Guide, Springer, 2nd edn., 2007, pp. 190-192.

39 W. Hu, C. Berdugo, and J. J. Chalmers, Cytotechnology, 2011, 63, 445-460.
40 M. J. Song, S. M. Brady-Kalnay, S. H. McBride, P. Phillips-Mason, D. Dean, and M. L. Knothe Tate, PLoS One, 2012, 7, e43601.

41 H. Bruus, Theoretical Microfluidics, Oxford University Press, USA, 2008, p346.

42 M. Mollet, R. Godoy-Silva, C. Berdugo, and J. J. Chalmers, Biotechnol. Bioeng., 2008, 100, 260-272. 